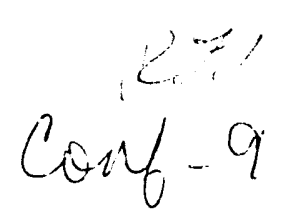

RFP- -4509

DE92 003637

\title{
LOW-COST TELEOPERATOR-CONTROLLED VEHICLE FOR DAMAGE ASSESSMENT AND RADIATION DOSE MEASUREMENT
}

\author{
W. H. Tyree \\ EG\&G Rocky Flats
}

\begin{abstract}
A low-cost, disposable, radio-controlled, remotereading, ionizing radiation and surveillance teleoperator re-entry vehicle has been built. The vehicle carries equipment, measures radiation levels, and evaluates building conditions.
\end{abstract}

The basic vehicle, radio control with amplifiers, telemetry, elevator, and video camera with monitor cost less than $\$ 2500$. Velcro-mounted alpha, beta-gamma, and neutron sensing equipment is used in the present system. Many types of health physics radiation measuring equipment may be substituted on the vehicle.

The system includes a black-and-white video camera to observe the environment surrounding the vehicle. The camera is mounted on a vertical elevator extendable to 11 feet above the floor. The present vehicle uses a video camera with an umbilical cord between the vehicle and the operators. Preferred operation would eliminate the umbilical. Video monitoring equipment is part of the operator control system.

Power for the vehicle equipment is carried on board and supplied by sealed lead-acid batteries. Radios are powered by $9-\mathrm{V}$ alkaline batteries. Battery supplies for locomotion, camera, and lights are separate to ensure well-defined vehicle battery capacity while the camera and lights are in use.

The radio control receiver, servo drivers, highpower amplifier and 49-MHz FM transceivers were irradiated at moderate rates with neutron and gamma doses to 3000 Rem and 300 Rem, respectively, to ensure system operation. These units performed normally during and after being exposed to these doses.

\section{INTRODUCTION}

One of the basic concepts in Health Physics radiation protection for industrial workers is the ALARA concept in the work place. That is, personnel radiation doses should be kept "As Low As Reasonably Achievable." This concept takes on paramount importance in an emergency condition where high radiation levels (or more importantly, unknown variable radiation levels) may be encountered. Presently, re-entry personnel may carry detector extension rods to minimize radiation dose. The rod has a maximum extension capability of about ten feet and provides a maximum dose reduction to the operator by a factor of 100 . To obtain this benefit, the operator must be strong enough to carry monitoring equipment with the detector extension, his own air supplies, and other equipment for dose assessment and personal safety.

Recent developments in mobile automatic machines have removed the need to use a human as an equipment carrier. These machines can easily carry sensing equipment that communicates with the human operator. This allows the operator more freedom to evaluate re-entry conditions. Many types of electronic equipment are available to implement the dose measuring and data trans-

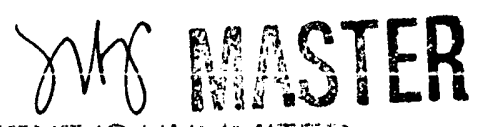

DIS TRIBUTION OF TIIS TOO MMENT IS UNLIMITEO 
mission systems mounted on a teleoperator vehicle. The basic feature of this teleoperator system uses the human as part of the controlling loop for interactive control.

With the operator 50 to 100 feet behind the vehicle, the operator dose may be as much as 10,000 times lower than that at the sensing detectors mounted on the vehicle.

The teleoperator system that I am about to describe is one of many ways to build a piece of equipment which will reduce the radiation dose to operating personnel involved in emergency operations. The primary concern in this paper is to describe the attitude for developin such a piece of equipment. I will show you what we developed using very ordinary equipment mostly from fields other than the nuclear instrument business. The area that I would like to explore is one of the development of the process by which you or your facility might build this kind of equipment.

The development of $\boldsymbol{\omega}$ new piece of equipment which has teleoperator capability should be looked at as an exercise in coordination of expertise from many technical fields. All too often, the development of a special remote controlled system is relegated to special groups. Of course they have special expertise, but they don't necessarily relate most effectively to the end user of the equipment. The end user gets a chance at operating the equipment only after it has been accepted by an engineering group, played with by another engineering group and finally passed to the emergency operations group for their use. The final user, sometimes, does nut recognize the equipment they asked for 2,3 or 4 years before. The point of all this is to have the engineering design team invite the user to be a part of the design team. The person on the floor is the one who will recognize that in an emergency, special parts of the operations are affected. The design engineer group rarely has any in-depth knowledge of the floor operations of the building. When the design group is being formed, look for people who have special backgrounds. In other words, the "ham" radio operator, the model airplane enthusiast, the person who lives in a wheel chair, the video recording buff. Name all of the special interest activities that are part of any group of people...they can contribute to the requirements and solutions for a teleoperator system which will be able to feed information to the facility operators and engineering personnel.

Pursue the normal path for obtaining special engineering input for the teleoperator system. The system may have to meet special radio frequency limits because of its use in secure areas. This may limit the frequencies of choice for pulse and video transmission. What special regulations are mandatory for system operation? Are there other ways to communicate to the remote system? Is an optical media possible? Each of these approaches will have its own problems to solve.

I have a good friend in the photography department who acts as a consultant on possible ways to take pictures both for immediate transmission and permanent recording for retrieval at a later time. Get to know the experts on special detectors that have equipment that might ride on this system. Do they already have equipment that could be used for a remote system? What does operations really want in the way of information? They will be the ones that recognize that the area has the wrong sound or the pipes are not in the right place.

Know who your friends are! These people are the jewels of your organization. They will be the ones that supply the seed money for the basic first level prototype. The opposite is just as important, know who your enemies are. Invite them to the design brainstorming sessions. Show 
them how the system will supply benefits to them, win them to your side. Try to develop or market your idea to middle management or higher. Get someone to support building a touchable prototype. People like to see what it is that they are buying. Proposals are a dime a dozen, so to speak. Tangible equipment, even if it is crude, sells the idea much faster than nebulous paper plans.

I have been a coach for an "Odyssey of the Mind"1 team for junior high school kids for the past several years. This program is a public school competition which gives a group of five students a creative problem to solve with a limited budget. The students must design, build, and present the solution as a district competition in the spring. At the beginning, some of the kids have never seen a screwdriver, but by the end of the project, they have carried a concept to a paper design and then to a final piece of hardware. In the process they have learned how to work as a team, create, compromise, and have deveioped a powerful familiarity with a $3 / 3$ inch power screwdriver.

I would like to propose that a teleoperator system be built with this type of approach used for the design team. Don't draw from all of the ordinary sources of equipment and materials. Use all of your resources, not just the specialists.

After the brainstorming has been completed, give the job to one person to coordinate. This person becomes the central figure in the process of building the transport system, determining the type of equipment on board, and letting the end user design the way the information is presented, in as meaningful way as possible. The more operations people are involved, the more likely the equipment will meet the original intent and the more likely the user will accept the equipment.

\section{Prototype Trials}

I have had the chance to take teleoperator systems to public school demonstrations, before they were put into plant use. It was very instructive to let elementary school kids operate a teleoperator system with an operational remote controlled TV camera on board. This is a test which is very much like passing a seismic qualification and mechanical reliability test at the same time. If the equipment can't survive the tests any good video game jockey can give a video game, the equipment isn't going to live up to a real emergency anyway. This is a very effective way to test your system for operation simplicity and ruggedness.

\section{On Board Equipment}

The system I am about to describe uses standard health physics monitoring equipment. The point is to use equipment which is part of the normal inventory and already in use in the facility. It will probably have up to date calibrations stickers and have been used routinely so it is likely to be operational.

Now, with all of these comments in mind, let's describe a mobile teleoperator system developed at Rocky Flats for re-entry use. This system cannot climb stairs, but the cost of the system is low enough so that one of these systems could be on each floor of a facility.

\section{BASIC VEHICLE}

The basic vehicle is constructed from a rebuilt electric wheelchair. Of the original chair configuration, only the wheels, belts, motors, and folding frame were retained. The " $\mathrm{X}$ " frame was fixed in position by bolting two plates to the top and bottom of the folding frame. These plates are 26 in. long by $16 \mathrm{in}$. wide by $1 / 8$ in. thick. 
The 24-V motors, mounted on each side of the frame, are an integral part of the vehicle. Motor power is set at $12-\mathrm{V}$. Other supply voltages were tried, but operator dexterity becomes critical when the power supply is above $18-\mathrm{V}$.

All control and power amplifiers to operate the vehicle drive motors are parts of the radio control system. The power amplifiers are connected through a filter to the motor winding. A description of the power amplifier is included in the Control Unit section.

\section{CONTROL UNIT}

The radio control unit was purchased from a local hobby shop handling radio control supplies. These controllers normally have an outside operational range of $1 / 4$ to $1 / 2$ mile. Inside buildings with large amounts of metal and reflecting surfaces, reliable ranges of about 200 feet have been obtained.

This system uses a Futaba radio control unit that has four-function control with outputs normally labelled for model plane operations including aileron and rudder. The two vertical axes control the motors of the wheelchair. The horizontal axes of the joy sticks control the elevator and tilt of the camera.

An FP-T4L and FR-R4f Futaba four-channel transmitter and receiver were used for radio control. Standard frequencies for radio control transmitter and receiver combinations for model boat and race cars are on 11 frequencies between 75.430 and $75.870 \mathrm{MHz}$.

Many types of radio control units are available. Some have as many as seven parameters available on the front panel of the control unit. Such units would allow additional options on the vehicle.
The power amplifier is a plug-in unit, which increases the normal current capability of the servo system operated by the receiver. Power amplifiers used on this vehicle are Futaba FPMC4-20. These units can control up to 20-A at $24-\mathrm{V}$. The input connections are plug compatible with the standard radio control receiver. The newer radio control units include internal parallel power MOSFETS which allow direct drive of high power motors.

\section{TELEMETRY}

Data from separate radiation instruments are handled by individual radio links operating on three different frequencies in the $49-\mathrm{MHz}$ band.

The transceiver has receiver sensitivity of $0.5 \mu \mathrm{V}$ minimum, image rejection of $20 \mathrm{~dB}$ minimum, and frequency stability of $\pm 2 \mathrm{kHz}$. The transmitter has an output of $10,000 \mu \mathrm{V}$ at $3 \mathrm{~m}$ (FCC maximum), spurious and harmonic emissions of $20 \mathrm{~dB}$ minimum, FM hum and noise of $40-\mathrm{dB}$ minimum, and frequency stability of $\pm 2 \mathrm{kHz}$.

Five frequencies between 49.830 and $49.890 \mathrm{MHz}$ are available in the $49-\mathrm{MHz}$ band. A pair of units is required to form a complete link from the vehicle to the operators. A specific frequency is selected for each of the alpha, beta-gamma, and neutron counters. Each radio operates with a standard $9-\mathrm{V}$ battery with $13.5 \mathrm{~mA}$ current drain.

These radios are modified with a miniature phone plug and cable in place of the normal microphone. The front panel sensitivity switch is set to the "LO" sensitivity position to minimize pulse distortion. Telemetry antennas are mounted on brackets with the transceiver unit clipped next to them. The shielded "mike" cable with instrument plug connects to the radiation measuring instrument audio output jack. A 
different frequency is selected for the alphaparticle, beta-gamma, and neutron instruments.

The $49 \mathrm{MHz}$ transceivers need to have the right plugs for the earphone connection to the transmitters. That means dedicating certain types of radiation equipment for use on the system, so that when you need it in a hurry, the equipment is compatible.

The video signal is connected by coaxial cable to a battery-powered black-and-white television monitor. Operators wear headsets that receive signals from the telemetry units transmitting alpha, beta-gamma, and neutron data. Separate audio signals are annunciated in separate earphones. The present system uses only the earphone output. The pulse rate heard by an experienced operator is interpreted as an approximate count rate.

\section{ELEVATOR}

An elevator is included on the vehicle to allow visual inspection with a video camera in the building overhead or as a movable platform for another radiation detector. The elevator uses two nested square shafts; the outside shaft is $11 / 4$ inch square aluminum channel, 6 feet long, having an inside U-shaped 1-inch-square aluminum channel with one side open. Stainless steel cable (480-lb test) connects the bottom of the channel inside the square shaft and runs up through a single pulley and down the outside of the elevator to a small $24-\mathrm{V}$ electric winch. The electric motor is controlled by the horizontal axis of a joy stick. The horizontal axis of the other joy stick controls the tilt of the elevator top. The top tilts from 75 degrees upward to 35 degrees downward.
-Figure One (Mobile Instr Transport Sys

\section{OPTIONAL FEATURES}

Figure 1 depicts a vehicle, system block diagram, a video color camera, and a $35-\mathrm{mm}$ camera. This system is envisioned as a means of obtaining "real time" visual information and high-quality pictures of inaccessible areas while assessing damage during initial re-entry.

The video camera signals are transmitted as radio frequency signals. A wide-band fast-scan TV signal would allow the operator complete freedom to operate the vehicle without the motion limitation of umbilical cables. A microphone enables personnel to listen to the noises present during re-entry and discern normal from abnormal sounds in specific areas.

Outputs of the three Maxon radio telemetry units are shown tied to digital scalers to obtain direct count information from each of the radiation sensor units. This presentation would allow an operator to gather count rate information directly from the on board counters. None of these options are incorporated into the present vehicle.

\section{RADIATION INSTRUMENTS}

The portable radiation instruments used for contamination control throughout the plant site provide basic detection functions for the vehicle.

Alpha particle detection uses a large area air proportional detector coupled to a multi-range ratemeter. The detector is mounted on the end of the vehicle to survey the floor ahead of the vehicle. A beta-gamma GM detector is placed on the top deck to monitor the beta-gamma field present around the vehicle. A portable version of the Rocky Flats criticality alarm detector is used for thermal neutron detection. The output 


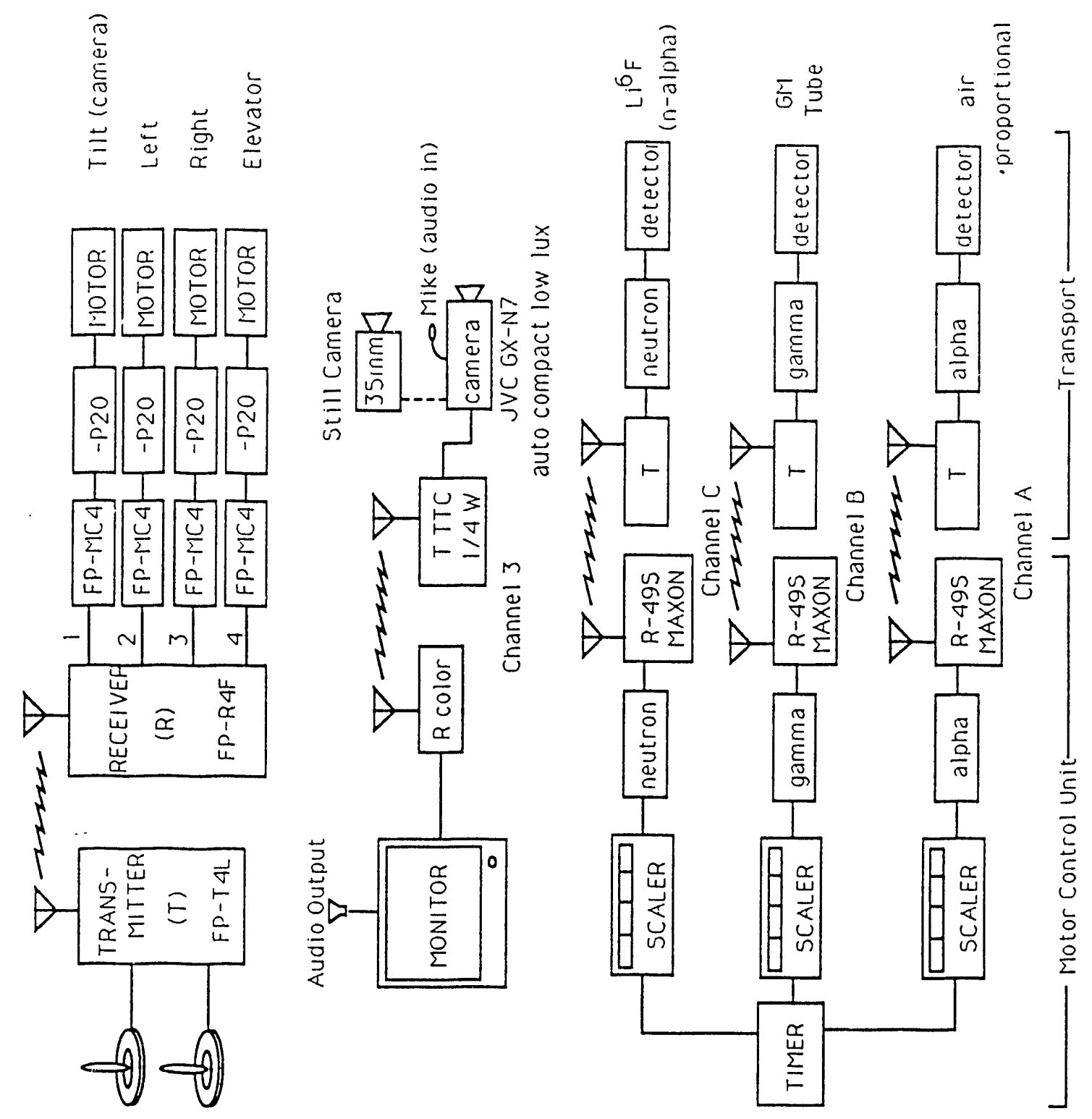


of each of these instruments pulse modulates a separate transceiver to produce a short audio pulse in the output of the receiver earphone. The speech options on new portable radiation instruments simplify the connection of the data output to the transceiver. The audio level directly feeds the low level microphone input. The operator gets direct counts per minute from the earphone.

The Velcro used for holding the instrument on the top of the vehicle can easily be attached to the bottom of the instrument cases of standard portable health physics equipment. Two inch wide Velcro is really nice stuff and does an amazing job of holding equipment. We tried holding a 4.85 pound instrument with 2 " Velcro and it easily passed a 6 Richter 2-axis shake test.

\section{RADIATION TESTS}

The equipment used in the telemetry and control of the transport and surveillance system was exposed to neutron and gamma radiation to determine performance under possible conditions present during an emergency.

The Maxon FM transceiver and the Futaba radio control and servo drive systems were exposed to a Californium-252 source for several weeks. Rate of exposure was about 6.4 Rem per hour for neutrons and $640 \mathrm{mRem}$ per hour for gamma. Each system was tested periodically throughout the exposure period. The Maxon transceivers were exposed to fields of 2000 Rem neutron and 200 Rem gamma. The Futaba receiver and servo amplifiers were exposed to 3000 Rem neutrons and 300 Rem gamma. All equipment operated normally during and after the tests.
The basic platform for the vehicle, two-wheel controller, amplifiers, and basic chair frame cost less than $\$ 1000$. Many accessories and instruments can abe added to the basic vehicle to provide optional features.

Itemized costs:

Basic wheelchair with motor, rebuilt standard size, no control system\$ 500

Futaba Radio Control Transmitter and Receiver 170

Futaba 20-A Amplifier, 4 ea. $\quad 396$ Maxon FM Transceivers, 3 ea. 264 Elevator Parts and Assembly 250 Camera, Black-and-White, Fixed Focus 600 Batteries, Sealed Lead Acid, 2 ea. 140 Lamps, Sealed Beam, 2 ea. 100

Total $\$ 2420$

\section{BATTERY POWER}

The vehicle and the camera system with lights are powered by separate sources. This guarantees that power available for controlling the vehicle is well defined. The $12-\mathrm{V}$ camera and lights consume most of the power.

Several types of batteries were used. Various types of gelled lead-acid batteries were tried but with limited success. Starved electrolyte leadacid types manufactured by Gates Rubber Company performed well. Sealed automotive batteries having very high current capability are now available on the automotive market. They are much lighter than the equivalent open-celled automotive wet cell. This type battery would ensure vehicle operation for more than one day's continuous use before recharging.

\section{costs}


The 9-V transistor radio batteries for the individual transceivers are mounted inside eacil unit.

\section{SUMMARY}

A low-cost teleoperator vehicle has been built. This vehicle includes various health physics monitoring instruments with low-cost fixed frequency radio links. A video camera is included on board for real-time visual information. The radio equipment for data transmission and vehicle control was tested in neutron and gamma fields to ensure operations in emergency condition.

The system provides re-entry personnel with a vehicle and sensors that assess the radiation and physical conditions present in a production building with minimum dose to the operators.

\section{NOTES}

1. C. Samuel Micklus, Odyssey of the Mind Program Handbook, Glassboro, N. J.: Creative Competition, Inc. 1988.

\section{BIBLIOGRAPHY}

1. A. J. Foltman (Ed.), Proceedings of the Workshop on Requirements of Mobile Teleoperators for Radiological Emergency Response and Recovery, June 24-25, 1985, ANL/EES-TM-291, Argonne National Laboratory.

2. Teleoperator Robotics in Hostile Environments, 1985, ISBN 0-87263-185-0, Robotics International, Society of Manufacturing Engineers.

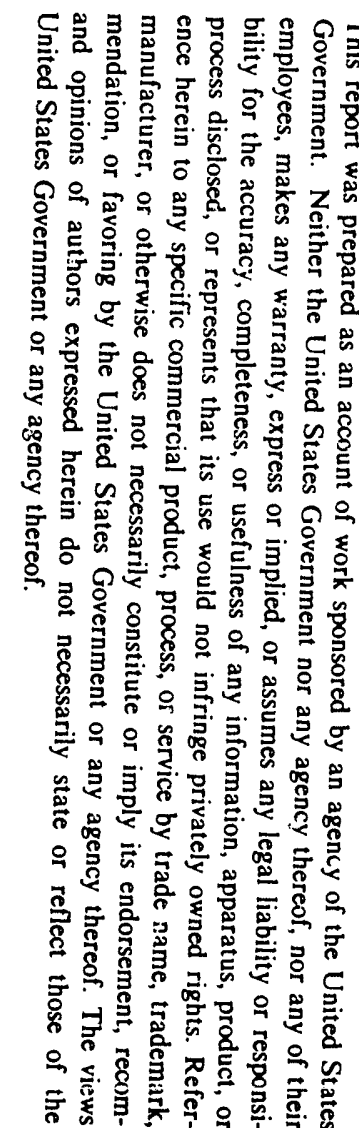



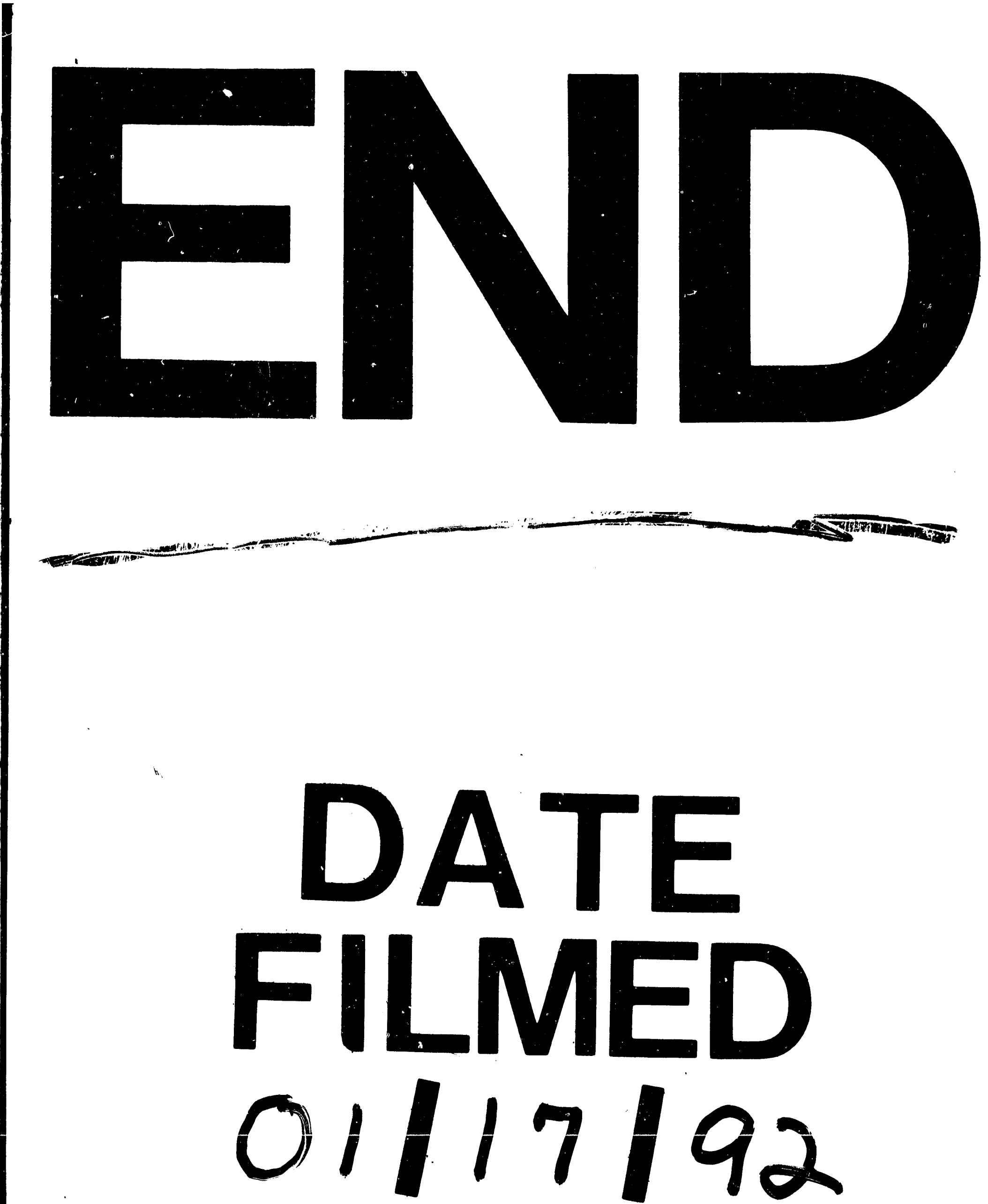
